\title{
Freezing stallion semen with the new Caceres extender improves post thaw sperm quality and diminishes stallion-to-stallion variability
}

\author{
A Morillo Rodriguez, C Ortega Ferrusola, B Macias Garcia, J M Morrell, \\ Heriberto Rodriguez-Martinez, J A Tapia and F J Pena
}

\section{Linköping University Post Print}

N.B.: When citing this work, cite the original article.

Original Publication:

A Morillo Rodriguez, C Ortega Ferrusola, B Macias Garcia, J M Morrell, Heriberto Rodriguez-Martinez, J A Tapia and F J Pena, Freezing stallion semen with the new Caceres extender improves post thaw sperm quality and diminishes stallion-to-stallion variability, 2011, Animal Reproduction Science, (127), 1-2, 78-83.

http://dx.doi.org/10.1016/j.anireprosci.2011.07.009

Copyright: Elsevier Masson http://www.elsevier-masson.fr/

Postprint available at: Linköping University Electronic Press http://urn.kb.se/resolve?urn=urn:nbn:se:liu:diva-72147 


\title{
Freezing Stallion semen with the new Cáceres ${ }^{\mathrm{TM}}$ extender improves post thaw sperm quality and diminishes stallion-to- stallion variability
}

Morillo Rodríguez A, Ortega Ferrusola C, Macías García B, ${ }^{2}$ Morrell JM, ${ }^{3}$ Rodríguez-Martínez

\author{
$H,{ }^{1}$ Tapia JA, Peña FJ
}

Laboratory of Equine Reproduction, Veterinary Teaching Hospital, ${ }^{1}$ Department of Physiology, Faculty of Veterinary Medicine, University of Extremadura, Avd. de la Universidad s/n Cáceres, Spain; ${ }^{2}$ Division of Reproduction, Faculty of Veterinary Medicine and Animal Sciences, Swedish University of Agricultural Sciences, Uppsala, Sweden; ${ }^{3}$ Department of Clinical and Experimental Medicine, Faculty of Health Sciences, Linköping University, Linköping, Sweden.

*Correspondence to Dr FJ Peña Veterinary Teaching Hospital, Laboratory of Equine Reproduction, Faculty of Veterinary Medicine University of Extremadura Avd de la Universidad s/n 10003 Cáceres Spain. E-mail fjuanpvega@unex.es

\section{Acknowledgements}

The investigations of the authors received financial support from Ministerio de Ciencia e Innovación- FEDER Madrid, Spain INIA RZ2008-00018-00-00, and AGL 2010-20758 GAN. The generous collaboration of the Service of Equine Breeding of the Spanish Army is greatly acknowledged.

\begin{abstract}
Split samples from 7 stallions were simultaneously frozen in four different extenders, INRA 96 egg yolk glycerol, Gent and the two newly developed extenders Caceres 1 and 2. After thawing, samples were evaluated for motility (CASA system) sperm membrane integrity, early membrane changes, acrosome integrity, and mitochondrial membrane potential (flow cytometry). Samples frozen in Caceres 2 extender consistently showed the best results in postthaw motility and velocity, membrane integrity and mitochondrial membrane potential. However, in terms of sperm motility, the results were not different from those observed in samples frozen in Cáceres 1 extender. It is concluded that these new extenders can be included in a freezeability test that the semen from each individual sire should undergo before entering a commercial freezing program.
\end{abstract}

Key words: stallion, frozen sperm, flow cytometry, CASA.

\section{INTRODUCTION}


Sperm-freezing technology is becoming an area of increasing interest for the equine industry (Samper, 2007), despite the technology yet being sub-optimal,mainly due to large inter-individual variability in sperm survival during the freezing and thawing procedures (Loomis and Graham 2002). This fact prevents the standardization of freezing and thawing protocols. Such variability is often ascribed to the selection done in horses, where most, if not all stallions have been chosen for reproduction only based in their performance and phenotype. The steady development of artificial insemination (AI) with frozen-thawed sperm in the equine industry requires efforts to improve the quality of frozen samples, and also to minimize individual variability among stallions in the freezeability of their ejaculates. Attempts to improve current cryopreservation protocols (Loomis and Graham, 2008, Vidament et al, 2000, 2005), and development of techniques for deep intrauterine insemination with a reduced number of spermatozoa (Lyle and Ferrer, 2005) are relatively recent innovations in equine breeding. In spite of these facts, cryopreservation protocols used in this species are still under development, mainly due to past limitations for AI in most of the stud books, and lack of funds for research.

Freezing and thawing cause major damage to spermatozoa, particularly their membranes (plasma- and organelle membranes) (Peña et al, 2003ab). Based on experimental studies of equine spermatozoa, the most important factors causing cryoinjury are considered to be the toxicity caused by unequal distribution of cryoprotectants (such as glycerol), and the osmotic stress caused by dehydration of the extender and the cells during freezing and again at thawing, disputing the findings of earlier -more empirical- studies (Morris et al, 2006, 2007). In addition, a number of other events occur during cooling: phase transitions in the plasmalemma, oxidative damage and premature ageing which all contribute to sperm death or, if surviving, to their shortened lifespan (Watson, 2000). Furthermore, apoptosis-like phenomena have been identified as taking place in spermatozoa of several species including horses (Martin et al, 2007; Martin et al, 2004; Ortega Ferrusola et al, 2008, 2009, Moran et al 2008), which explains not only cellular death, but also the different degrees of subtle cellular damage that most surviving spermatozoa disclose post-thaw.

In view of these facts, the aim of this study was to test the efficacy of a recently developed extender for stallion sperm, designed to reduce the osmotic stress during freezing and thawing, and compare it with some of the currently available commercial extenders for the equine species.

\section{Material and methods}

\section{Semen collection and processing}

Semen (four ejaculates per stallion) was obtained from 7 Pure Spanish stallions (PRE) individually housed at the Veterinary Teaching Hospital of the University of Extremadura, Cáceres, Spain. The stallions were maintained according to institutional and European regulations, with semen being collected on a regular basis (two collections/week) during the 2009 breeding season. Ejaculates were collected using a Missouri model artificial vagina, lubricated and pre-warmed to $45-50^{\circ} \mathrm{C}$, fitted with an in line filter to separate the gel fraction. The collected ejaculate was immediately transported to the laboratory for evaluation and processing. The filtered ejaculate was extended 1:1 (v/v) with INRA 96 (IMV, L 'Aigle, France), split in four subsamples and centrifuged at $600 \mathrm{~g}$ for 10 minutes. The resulting sperm pellets were re-extended in four different freezing media (i) Gent, (Minitüb Ibérica, Spain), (ii) INRA 96 (IMV, L `Aigle, France), supplemented with 2\% egg yolk and 2,5\% glycerol, 
(iii) Cáceres 1 and (iv) Cáceres 2 (University of Extremadura, Cáceres Spain, patent pending), to a final concentration of $100 \times 10^{6}$ spermatozoa per $\mathrm{mL}$. The spermatozoa were slowly cooled to $4^{\circ} \mathrm{C}$ within one hour, loaded in $0.5 \mathrm{~mL}$ plastic straws and frozen horizontally in racks placed $4 \mathrm{~cm}$ above the surface of $\mathrm{LN}_{2}$ for $10 \mathrm{~min}$, after which they were directly plunged in $\mathrm{LN}_{2}$ for storage. After at least 4 weeks of storage, straws were thawed in a water bath at $37^{\circ} \mathrm{C}$ for 30 seconds, for analyses.

\section{Sperm motility analysis}

Motility and sperm kinematics were evaluated after thawing and after incubation at $38^{\circ} \mathrm{C}$ for 60 minutes (thermoresistance test). Motility was measured using a CASA system (ISAS ${ }^{\circledR}$ Proiser Valencia Spain) based on the examination of 25 consecutive digitalized images obtained from a single field using an $\mathrm{x} 10$ negative phase contrast objective in a light microscope (Olimpus CX41 Tokyo, Japan). A minimum of 200 cells per sample was analyzed. Images were taken with a time lapse of $1 \mathrm{sec}$; the image capture speed was, therefore, one every $40 \mathrm{~ms}$. The number of objects incorrectly identified as spermatozoa was minimized on the monitor by using the playback function. With respect to the setting parameters for the program, spermatozoa with a VAP $<10 \mu \mathrm{m} / \mathrm{sec}$ were considered immotile, while spermatozoa with a velocity $>15 \mu \mathrm{m} / \mathrm{sec}$ were considered motile. Spermatozoa deviating $<45^{\circ}$ from a straight line were designated as linearly motile.

\section{Evaluation of acrosomal status}

The acrosomal status of sperm was evaluated as described by Rathi et al (2001) for stallion spermatozoa by staining the incubated samples with $5 \mu \mathrm{g} / \mathrm{ml}$ FITC-PNA $(100 \mu \mathrm{g} / \mathrm{ml}$ in PBS) (Sigma Aldrich St Louis Mo) and one $\mu \mathrm{L}$ of Ethidium Homodimer-1 (1.167 mM) (Molecular Probes Europe, Leiden, The Netherlands). The samples were incubated in the dark for 30 minutes at $38^{\circ} \mathrm{C}$ and assessed by flow cytometry and confocal laser microscopy. FITC -PAN staining differentiated in the viable sperm population (Eth-1 negative) two distinct subgroups. The first with intact acrosomes (not stained by the PNA) and those showing acrosomal FICTPNA staining which indicates that their acrosome are reacted or had reacted (Rathi et a 2001).

\section{Evaluation of mitochondrial membrane potential ( $\Delta \Psi m)$}

The lipophilic cationic compound 5,5',6,6'-tetrachloro-1,1',3,3' tetraethylbenzymidazolyl carbocianyne iodine (JC-1, Molecular Probes Europe, Leiden, The Netherlands) has the unique ability to label differentially mitochondria with low and high membrane potential. In mitochondria with high membrane potential, JC-1 forms multimeric aggregates emitting in the high orange wavelength of $590 \mathrm{~nm}$, when excited at $488 \mathrm{~nm}$. However at the same excitement wavelength $(488 \mathrm{~nm})$ in mitochondria with low membrane potential, the JC-1 forms monomers, which emit in the green wavelength $(525$ to $530 \mathrm{~nm})$. For staining, a $3 \mathrm{mM}$ stock solution of JC-1 was prepared in DMSO. From each sperm suspension, $5 \times 10^{6}$ freshly ejaculated or post-thawed spermatozoa were placed in one $\mathrm{mL}$ of PBS and stained with 0.5 $\mu \mathrm{L}$ JC-1 stock solution. The samples were incubated at $38{ }^{\circ} \mathrm{C}$ in the dark for 40 minutes before flow cytometric analysis. 
Early sperm membrane changes and viability were determined as described by Peña et al. (2005), with modifications for adaptation to the equine species (Ortega Ferrusola et al, 2008). In brief, one $\mathrm{mL}$ of sperm suspension $\left(5 \times 10^{6}\right.$ spermatozoa/mL) from freshly ejaculated or post-thawed spermatozoa was loaded with $3 \mu \mathrm{L}$ of YO-PRO-1 $(25 \mu \mathrm{M})$ and one $\mu \mathrm{L}$ of Ethidium Homodimer-1 (1.167 mM) (Molecular Probes Europe, Leiden, The Netherlands). After thorough mixing the suspension was incubated at $37^{\circ} \mathrm{C}$ in the dark for $16 \mathrm{~min}$. This staining distinguishes four sperm subpopulations. The first is the subpopulation of unstained spermatozoa. These spermatozoa are considered alive and without any membrane alteration. Another sperm subpopulation consists of YO-PRO-1 positive cells emitting green fluorescence. It has been demonstrated that in the early stages of apoptosis there is a modification of membrane permeability that selectively allows entry of some non-permeable DNA-binding molecules (Ormerod et al, 1993; Wronsky et al, 2002). This subpopulation contains spermatozoa which may show early damage or a shift to another physiological state, since membranes become slightly permeable during the first steps of cryoinjury, enabling YO-PRO-1 but not ethidium homodimer to penetrate the plasma membrane (Idziorek et al, 1995; Wronsky et al, 2002). Neither of these probes enters intact cells. Finally, two subpopulations of cryo-induced necrotic spermatozoa were easily detected, early necrotic, spermatozoa stained both with YO-PRO-1 and ethidium homodimer (emitting both green and red fluorescence), and late necrotic spermatozoa, cells stained only with ethidium homodimer (emitting red fluorescence).

\section{Flow Cytometry}

Flow cytometric analyses were carried out with a Coulter EPICS XL (Coulter Corporation Inc., Miami, FL, USA) flow cytometer equipped with standard optics, an argon-ion laser (Cyonics, Coherent, Santa Clara, CA, USA) performing $15 \mathrm{~mW}$ at $488 \mathrm{~nm}$ and an EXPO 2000 software. Subpopulations were divided by quadrants, and the frequency of each subpopulation was quantified. Non-sperm events (debris) were gated out based on the forward scatter and side scatter dot-plots by drawing a region enclosing the cell population of interest. Events with scatter characteristics similar to spermatozoa but without reasonable DNA content were also gated out. Forward and sideways light scatter were recorded for a total of 10,000 events per sample (YO-PRO-1, PNA), or 30,000 events for JC-1. Samples were measured at flow rate of 200-300 cells/sec. Green fluorescence was detected in FL1 red fluorescence was detected in FL3, and orange fluorescence in FL2.

\section{Confocal Microscopy}

Sperm samples were observed under a spectral inverted confocal microscope (Bio Rad MRC 1024, Hercules CA USA). The localization of each probe emission was assessed at a wavelength range of 495-545nm and 580-620 nm. Samples were excited with an argon ion laser emitting at $488 \mathrm{~nm}$.

\section{Statistical analysis}

The data were first examined using the Kolmogorov-Smirnov test to determine their distribution. If non-Gaussian distribution of some of the data gathered were detected after multivariate analysis of variance was performed, and when significant differences were found, the non-parametric Mann-Whitney $U$-test was used to compare pairs of values 
directly. All analyses were performed using SPSS ver. 15.0 for Windows (SPSS Inc., Chicago, IL). Statistical significance was set at $P<0.05$.

\section{RESULTS}

\section{Sperm motility and kinematics post thaw}

Motility was higher in the samples frozen using the Cáceres extenders (table 1). The percentage of motile sperm increased $(\mathrm{p}<0.05)$ by $11 \%$ and $17 \%$ when the extender Cáceres 2 was compared with the INRA96EYG and Gent extenders, respectively. After 60 minutes of incubation, the same tendency persisted (table 2). Sperm velocities were also higher in samples frozen in the two Cáceres extenders, except for VSL at thawing. However, after 60 minutes of incubation at $38^{\circ} \mathrm{C}$, all sperm velocities were higher in samples frozen in the Cáceres extenders. Interestingly, the lowest variability was observed in samples frozen in both Cáceres extenders. On the other hand, samples frozen in Gent extender showed the greatest variability with stallions depicting motilities post thaw from 7.5 to $52 \%$.

\section{Sperm viability and early changes in sperm membranes post thaw}

The percentage of intact sperm membranes post thaw was higher in samples frozen in Cáceres 2 extender, while there where no differences in the percentage of sperm membranes between the samples frozen in Gent and INRA96EYG extenders (table 3). Also the percentages of late apoptotic and necrotic spermatozoa were lower in samples frozen in both Cáceres extenders.

\section{Acrosomal status post thaw}

The percentage of live sperm with intact acrosomes post thaw was significantly higher $(\mathrm{p}<$ 0.05) in samples frozen in both Cáceres extenders, with increases of $14 \%$ when compared with samples frozen in Gent extender and $11 \%$ when compared with INRA96EYG extender (Table 4).

\section{Mitocondrial membrane potential}

The best results after freezing, in terms of percentage of active mitochondria post thaw, were observed in samples frozen in Cáceres 2 extender (table 5); i.e. sperm frozen in this extender had more active mitochondria.

\section{DISCUSSION}

In the present study, stallion ejaculates frozen in the extender Cáceres2 survived better freezing and thawing than semen samples frozen in commercial extenders currently available. In addition, stallion -to- stallion variability was lower in samples frozen in both Cáceres extenders.

Most damage during the cryopreservation process in the equine species is caused by osmotic stress, mainly at thawing (Morris 2007). The greater sperm survival of the samples frozen in Caceres extenders can be attributable to a reduction in the osmotic stress, due to the combination of cryoprotectants used in these extenders. These extenders use different amides (Chalah et al, 1999, Alvarenga et al, 2005) in combination with a lower dose of glycerol, thus 
diminishing glycerol toxicity, alongside with a reduction of osmotic shock due to the lower molecular weight of amides and their higher membrane permeability (Vidament et al, 2009).

One aspect of interest is that both extenders improved sperm survival in all tested stallions, while the other extenders presented greater variability in terms of sperm survival. Only in one stallion, showed Gent extender results comparable to the Cáceres 2 extender. This confirms previous observations, indicating that the design of stallion specific freezing protocols and or extenders is the best approach to improve results of frozen semen in the equine industry (Loomis and Graham, 2008).

Sperm frozen in Cáceres 2 extender improved all parameters of sperm quality studied. This aspect is of great importance considering most studies in horses only evaluate sperm motility post thaw. Moreover, the sperm plasma membrane serves as the main physical barrier to the outside environment and it is a primary site for freeze-thaw damage (Ricker et al, 2006). Recent research has revealed the sperm mitochondria as key structures for sperm function (see Peña et al, 2009 for a review). Special points of interest are that in humans, high mitochondrial potential $(\Delta \Psi \mathrm{m})$ is highly correlated with fertility capability (Gallon et al, 2006) and that the sperm mitochondria are those organelles of the stallion spermatozoon more sensitive to cold shock (Ortega Ferrusola et al, 2008). In view of this evidence, we paid special attention to the evaluation of the sperm membranes (plasma and acrosome membranes) and mitochondria. We used two approaches to study sperm membranes, firstly studied intact membranes (plasma and acrosome membranes), and also studied the integrity of the sperm membrane and early changes in their permeability, a change associated with sub lethal damage (Pena et al, 2004, Ortega Ferrusola et al, 2008, 2009, 2010). When the percentage of completely intact sperm membranes was considered, the best results were those of samples frozen in the Caceres 2 extender, while; when considering percentage of live sperm with intact acrosomes, both Caceres 1 and 2 extenders gave the best results. Apparently, freezing sperm in the Caceres 2 prevents better those early damages on sperm membranes, since while there was no difference in terms of ethidium (non permeable probe) exclusion. When ethidium was combined with YO-PRO-1, a semipermeable probe, Caceres 2 gave more live spermatozoa.

The sperm mitochondria is becoming a central assay of the evaluation of the spermatozoa since recent research demonstrate a pivotal role for them in the fertilizing potential of spermatozoa (Gallon et al, 2006). In term of the functionality of the mitochondria, samples frozen in extender Caceres 2 gave the best results. These results can also be attributable to a reduction of the osmotic shoc: Recent research in our laboratory (Macías García et al, submitted) demonstrated the greater susceptibility to osmotic-induced stress on mitochondria. Mitochondrial dysfunction appeared earlier than plasma membrane damage, indicating greater susceptibility to osmotic stress. The protective effect of Caceres 2 extender on stallion sperm mitochondria may indicate that in samples frozen in this extender there are more spermatozoa with high fertilizing potential (Gallon et al 2006).

Another important aspect that should be stressed is that there was a great variability in the response to freezing in the Gent extender, this extender gave results comparable to Caceres 2 in stallion number one, but very poor results in other stallions. This fact indicates, as previously stated (Loomis and Graham, 2008) the need to perform customized protocols for every independent stallion. Interstingly, Caceres 2 gave consistent results with all the stallions tested in this study.

In sum, newly designed extenders improved sperm quality post thaw, and diminished stallion-to-stallion variability. These extenders should be included in the pre-freeze test for 
every individual stallion in order to increase the number of stallions included in commercial freezing programs.

\section{REFERENCES}

Alvarenga MA, Papa FO, Landim Alvarenga FC, Medeiros AS Amides as cryoprotectants for freezing stallion sperm: a review Anim Reprod Sci 2005; 89: 105-113.

Carmo MT, Papa FO, Medeiros AS, Araujo GH, Alvarenga MA Improvement of stallion semen post thaw motility with the association dimethylformamide and methyl formamide as cryoprotectors Anim Reprod Sci 2005; 89:286-288.

Chalah T, Seigneurin F, Blesbois E, Brillard JP In vitro comparison of fowl sperm viability in ejaculates frozen by three different techniques and relationship with subsequent fertility in vivo Cryobiology 1999; 39: 185-191.

Ecott P, Viadament M, deMornac A, Perigault K, Climent F, Palmer E Freezing of stallion semen: interactions among cooling treatments, semen extenders and stallions J Reprod Fertil Suppl 2000; 56: 141-150.

Gallon F, Marchetti C, Jouy N, Marchetti Ph The functionality of the mitochondria differenciates human spermatozoa with high and low fertilizing capability Fertil Steril 2006; 86: $1526-1530$.

Idziorek T, Estaquier J, de Bels F, Ameisen JC. YOPRO-1 permits cytofluorometric analysis of programmed cell dead (apoptosis) without interfering with cell viability $\mathrm{J}$ Immunol Meth 1995; 185: 249-85.

Loomis PR, Graham JK 2008 Commercial semen freezing : Individual male variation in cryosurvival and the response of stallion sperm to customized freezing protocols Anim Reprod Sci 105: 119-128

Lyle SK, Ferrer MS Low dose insemination why when and how Theriogenology 2005; 64: 572-579.

Macías García B, Miro Moran A, Gonzalez Fernandez L, Ortega Ferrusola C, Morillo Rodriguez A, Gallardo Bolaños JM, Balao da Silva C, Rodriguez Martínez H, Tapia JA Peña FJ The mitocondria of stallion spermatozoa are more sensitive than the plasmalemma to osmotic induced stress: role of c jun NHP2 terminal kinases (JNKs) SPAKs (submitted)

Martin G, Cagnon N, Sabido O, Sion B, Grizard G, Durand P, Levy R (2007) Kinetics of occurrence of some features of apoptosis during the cryopreservation process of bovine spermatozoa Human Reproduction 2007; 22: 380-388

Martin G, Sabido O, Durand P, Levy R- Cryopreservation induces an apoptosis like mechanism in bull sperm Biology of Reproduction 2004; 71: 28-37 
Morán JM, Madejón L, Ortega Ferrusola C Peña FJ (2008) Nitric oxide induces caspase activity in boar spermatozoa Theriogenology 70: $91-96$

Morris GJ 2006 Rapidly cooled human sperm: no evidence of intracellular ice formation Human Reproduction 21: 2075-2083.

Morris, G.J., Faszer, K., Green, J.E., Draper, D., Grout, B.W.W., Fonseca, F., 2007. Rapidly cooled horse spermatozoa: loss of viability is due to osmotic imbalance during thawing, not intracellular ice formation. Theriogenology $68,804-812$.

Ormerod MG, Sun XM, Snowden RT, Davies R, Fearnhead H, Cohen GM. Increased membrane permeability of apoptotic thymocites: a flow cytometric study. Cytometry 1993; 14: 595-02

Ortega- Ferrusola C , Sotillo-Galán Y, Varela -Fernández E, Gallardo-Bolaños JM, González-Fernández L, Tapia JA, Peña FJ (2008) Detection of apoptosis like changes during the cryopreservation process in equine sperm Journal of Andrology 29:213-221.

Ortega- Ferrusola C , Sotillo-Galán Y, Varela -Fernández E, Gallardo-Bolaños JM, González-Fernández L, Tapia JA, Rodríguez Martinez H, Peña FJ (2009) Apoptotic markers can be used to forecast the freezeability of stallion spermatozoa Animal Reproduction Science 114: 393-403.

Ortega Ferrusola C, González Fernández L, García Macías B, Salazar Sandoval C, Morillo Rodríguez A, Rodríguez Martinez H, Tapia JA, Peña FJ (2009). Effect of cryopreservation on nitric oxide production in stallion spermatozoa Biology of Reproduction 81: 1106-1111.

Ortega Ferrusola C, González Fernández L, García Macías B, Salazar Sandoval C, Morell JM, Rodríguez Martinez H, Tapia JA, Peña FJ. (2009) Lipid peroxidation, assessed with BODIPY-C11, increases after cryopreservation of stallion spermatozoa is stallion dependent and relates to "apoptosis like" changes. Reproduction 138: 55-63.

Peña FJ' Johannisson A, Wallgren M, Rodriguez Martinez H. Antioxidant supplementation of boar spermatozoa from different fractions of the ejaculate improves cryopreservation: changes in sperm membrane lipid architecture Zygote 2004; 12:117-124.

Peña FJ' Johannisson A, Wallgren M, Rodriguez Martinez H. Antioxidant supplementation of boar spermatozoa from different fractions of the ejaculate improves cryopreservation: changes in sperm membrane lipid architecture Zygote 2004; 12:117-124.

Peña FJ' Johannisson A, Wallgren M, Rodriguez Martinez H. Assessment of fresh and frozen-thawed boar semen using an annexin-v assay: a new method of evaluating sperm membrane integrity. Theriogenology 2003; 60: 677-689.

Peña FJ' Saravia F, Johannisson A, Wallgren M, Rodriguez Martinez H. A new and simple method to evaluate early membrane changes in frozen-thawed boar spermatozoa International Journal of Andrology 2005; 28: 107-114. 
Peña FJ, Rodríguez Martínez H, Tapia JA, Ortega Ferrusola C, Fernández González L, Macias García B (2009) Mitochondria in mammalian sperm physiology and pathology: a review Reproduction in Domestic Animals 44: 345-349.

Rathi R, Colembrander B, Bevers MM, Gadella BM Evaluation of in vitro capacitation of stallion spermatozoa Biol Reprod 2001; 65:462-470

Ricker JV, Linfor JL, Delfino WJ, Kysar P, Scholtz EL, Tablin F, Crowe JH, Ball BA, Meyers SA Equine sperm membrane phase behaviour: the effects of lipid based cryoprotectants Biol Reprod 2006; 74:359-365.

Samper JC, Estrada AJ, McKinnon AO (2007) Insemination with frozen semen In Samper JC, Pycock JF, MacKinnon AO (eds) Current Therapy in Equine Reproduction

Saunders Elsevier St Luois Missouri USA pp 285-289

Vidament M French field results (1985-2005) on factors affecting fertility of frozen stallion semen Anim Reprod Sci 2005; 89: 115-136

Vidament M, Ecot P, Noue P, Bourgeois C, Magistrini M, Palmer E Centrifugation and addition of glycerol at 22 degrees instead of 4 degrees improve post thaw motility and fertility of stallion spermatozoa Theriogenology 2000; 54:907-919.

Vidament M, Vincent P, Martin FX, Magistrini M, Blesbois E Differences in ability of jennies and mares to conceive with cooled and frozen seen containing glycerol or not Anim Reprod Sci 2009; 112: 22-35.

Watson PF. The causes of reduced fertility with cryopreserved semen Anim Reprod Sci 2000; 60-61: 481-92

Wronsky R, Golob N, Grygar, E, Winsdish, M. Two color, fluorescence -based microplate assay for apoptosis detection Biotechniques 2002; 32: 666-68 
Table 1.- Motility (Computer assisted sperm analysis, CASA) at thawing of ejaculates from 7 stallions split and frozen in four different extenders (means \pm SD). Experiments were repeated four times for each stallion.

\begin{tabular}{lllll}
\hline & Caceres 1 & Caceres 2 & Ghent & INRA96EYG \\
\hline TM\% & $34.0 \pm 11.53^{\mathrm{a}, \mathrm{b}}$ & $40.4 \pm 14.67^{\mathrm{a}}$ & $28.8 \pm 19.71^{\mathrm{b}}$ & $23.5 \pm 12.39^{\mathrm{b}}$ \\
LM\% & $10.4 \pm 4.52^{\mathrm{a}}$ & $12.2 \pm 6.88^{\mathrm{a}}$ & $8.5 \pm 8.92^{\mathrm{a}}$ & $5.5 \pm 4.52^{\mathrm{b}}$ \\
VCL $(\mu \mathrm{m} / \mathrm{s})$ & $40.3 \pm 5.52^{\mathrm{a}}$ & $37.5 \pm 7.40^{\mathrm{a}, \mathrm{b}}$ & $35.0 \pm 9.67^{\mathrm{a}, \mathrm{b}}$ & $32.5 \pm 6.75^{\mathrm{b}}$ \\
VAP $(\mu \mathrm{m} / \mathrm{s})$ & $21.7 \pm 2.79^{\mathrm{a}}$ & $20.4 \pm 4.17^{\mathrm{a}, \mathrm{b}}$ & $18.9 \pm 4.98^{\mathrm{a}, \mathrm{b}}$ & $17.5 \pm 3.49^{\mathrm{b}}$ \\
VSL $(\mu \mathrm{m} / \mathrm{s})$ & $15.6 \pm 2.67$ & $15.0 \pm 3.54$ & $13.6 \pm 4.70$ & $12.9 \pm 3.30$ \\
\hline
\end{tabular}

$\mathrm{a}-\mathrm{b}$ within a row values with different superscript are statistically different $(\mathrm{P}<0.05)$

TM\% percentage of total sperm motility post thaw, LM\% percentage of linearly motile sperm post thaw, VCL circular velocity, VAP, average velocity, VSL, straight line velocity.

Table 2. Motility (Computer assisted sperm analysis, CASA) after incubation at $38^{\circ} \mathrm{C}$ for 60 minutes of ejaculates from 7 stallions split and frozen in four different extenders (means \pm $\mathrm{SD})$. Experiments were repeated four times for each stallion.

\begin{tabular}{lllll}
\hline & Caceres1 & Caceres2 & Ghent & INRA96EYG \\
\hline TM\% & $30.5 \pm 9.47^{\mathrm{a}, \mathrm{b}}$ & $32.7 \pm 14.44^{\mathrm{a}}$ & $21.9 \pm 17.26^{\mathrm{a}, \mathrm{b}}$ & $18.3 \pm 9.61^{\mathrm{b}}$ \\
LM\% & $8.63 \pm 3.90^{\mathrm{a}}$ & $8.8 \pm 6.04^{\mathrm{a}}$ & $4.6 \pm 6.32^{\mathrm{b}}$ & $2.5 \pm 2.17^{\mathrm{b}}$ \\
VCL $(\mu \mathrm{m} / \mathrm{s})$ & $36.5 \pm 6.40^{\mathrm{a}}$ & $34.1 \pm 6.57^{\mathrm{a}, \mathrm{c}}$ & $29.3 \pm 8.60^{\mathrm{b}, \mathrm{c}}$ & $27.5 \pm 5.01^{\mathrm{b}}$ \\
VAP $(\mu \mathrm{m} / \mathrm{s})$ & $20.0 \pm 3.63 \mathrm{a}$ & $18.6 \pm 3.64 \mathrm{a}$ & $15.6 \pm 4.90 \mathrm{~b}$ & $14.7 \pm 2.83 \mathrm{~b}$ \\
VSL $(\mu \mathrm{m} / \mathrm{s})$ & $14.2 \pm 3.46^{\mathrm{a}}$ & $14.0 \pm 3.22^{\mathrm{a}}$ & $10.9 \pm 4.66^{\mathrm{b}}$ & $10.4 \pm 2.77^{\mathrm{b}}$ \\
\hline
\end{tabular}

a-b within a row values with different superscript are statistically different $(\mathrm{P}<0.05)$ $\mathrm{a}-\mathrm{b}$ within a row values with different superscript are statistically different $(\mathrm{P}<0.05)$ TM\% percentage of total sperm motility post thaw, LM\% percentage of linearly motile sperm post thaw, VCL circular velocity, VAP, average velocity, VSL, straight line velocity. 
Table 3. Membrane integrity and sub lethal damage after thawing minutes of ejaculates from 7 stallions split and frozen in four different extenders (means \pm SD). Experiments were repeated four times for each stallion.

\begin{tabular}{lllll}
\hline & Cáceres1 & Cáceres2 & Ghent & INRA96EYG \\
\hline Live & $33.6 \pm 10.05^{\mathrm{a}}$ & $40.4 \pm 8.92^{\mathrm{b}}$ & $29.2 \pm 10.11^{\mathrm{a}}$ & $29.0 \pm 8.91^{\mathrm{a}}$ \\
YoPro+ & $18.1 \pm 8.03^{\mathrm{a}}$ & $12.3 \pm 4.77^{\mathrm{b}}$ & $9.8 \pm 6.49^{\mathrm{ab}}$ & $12.6 \pm 7.48^{\mathrm{ab}}$ \\
YoPro+Eth+ & $16.3 \pm 6.66^{\mathrm{a}}{ }^{\mathrm{b}}$ & $12.5 \pm 4.53^{\mathrm{b}}$ & $17.9 \pm 9.81^{\mathrm{a}}$ & $19.3 \pm 10.06^{\mathrm{a}}$ \\
Eth+ & $31.9 \pm 10.70^{\mathrm{a}}$ & $34.7 \pm 8.88^{\mathrm{a}}$ & $43.1 \pm 12.03^{\mathrm{b}}$ & $39.07 \pm 13.05^{\mathrm{ab}}$ \\
\hline
\end{tabular}

$\mathrm{a}-\mathrm{b}$ within a row values with different superscript are statistically different $(\mathrm{P}<0.05)$ Live $\%$ of intact membranes, YoPro+, percentage of spermatozoa with increased membrane permeability (indicative of sublethal damage), YOPRO+/Eth + and Eth + are dead spermatozoa.

Table 4. Membrane and acrosomal status post thaw of ejaculates from 7 stallions split and frozen in four different extenders (means $\pm \mathrm{SD}$ ). Experiments were repeated four times for each stallion.

\begin{tabular}{lllll}
\hline & Caceres1 & Cáceres2 & Ghent & INRA96EYG \\
\hline PNA-/Eth- & $47.6 \pm 9.55^{\mathrm{a}}$ & $48.0 \pm 10.59^{\mathrm{a}}$ & $34.1 \pm 10.96^{\mathrm{b}}$ & $37.6 \pm 9.21^{\mathrm{b}}$ \\
PNA+ & $0.5 \pm 0.84$ & $1.5 \pm 3.37$ & $2.5 \pm 8.68$ & $0.4 \pm 0.48$ \\
PNA+/Eth+ & $5.4 \pm 6.32$ & $7.8 \pm 10.20$ & $10.6 \pm 9.13$ & $4.9 \pm 4.9$ \\
Eth+ & $46.6 \pm 7.22^{\mathrm{a}}$ & $42.6 \pm 7.22^{\mathrm{a}, \mathrm{b}}$ & $52.7 \pm 12.8^{\mathrm{b}}$ & $57.1 \pm 8.84^{\mathrm{c}}$ \\
\hline
\end{tabular}

a-c within a row values with different superscript are statistically different $(\mathrm{P}<0.05)$

PNA-/Eth-, \% intact membrane and intact acrosomes, PNA+ \% intact membranes and damaged acrosomes, PNA+/Eth+ $\%$ dead sperm with damaged acrosomes, Eth + percentage of dead sperm. 
Table 5. Mitochondrial membrane potential $\Delta \Psi \mathrm{m}$ of ejaculates from 7 stallions split and frozen in four different extenders (means $\pm \mathrm{SD}$ ). Experiments were repeated four times for each stallion.

\begin{tabular}{lllll}
\hline & Cáceres 1 & Caceres 2 & Ghent & INRA96EYG \\
\hline High $\Delta \Psi \mathrm{m}$ & $3.4 \pm 5.40$ & $5.2 \pm 4.07$ & $3.0 \pm 3.38$ & $2.1 \pm 1.77$ \\
High and Low $\Delta \Psi \mathrm{m}$ & $17.7 \pm 9.31^{\mathrm{a}}$ & $29.7 \pm 8.57^{\mathrm{b}}$ & $20.4 \pm 10.67^{\mathrm{a}}$ & $19.4 \pm 7.67^{\mathrm{a}}$ \\
Low $\Delta \Psi \mathrm{m}$ & $78.3 \pm 12.30^{\mathrm{c}}$ & $64.6 \pm 9.88^{\mathrm{d}}$ & $76.1 \pm 11.69^{\mathrm{c}}$ & $78.0 \pm 11.92^{\mathrm{c}}$ \\
\hline
\end{tabular}

a-d within a row values with different superscript are statistically different $(a, b \quad P<0.05$; $c-d$ $\mathrm{p}<0.01)$

High $\Delta \Psi \mathrm{m}, \%$ of spermatozoa with all their mitochondrial showing high potential, High and low $\Delta \Psi \mathrm{m}$, percentage of spermatozoa depicting simultaneously mitochondrial with high al low potential, Low $\Delta \Psi \mathrm{m}$, percentage of spermatozoa with all their mitochondria depicting low potential. 